some length in more than one institution. I believe, however, that "sufficient evidence is seldom available at the time of referral to justify an individual psychiatrist recommending detention of a patient." 1 In addition, I would quote Courtney Wade's perceptive comment: "The psychiatrist, however, can treat the patient only on the findings and the history. Where the history is a fabrication, then the findings and treatment are based on false premises."'2

A schizophrenic once challenged me with the following couplet:

You want to get inside my head and see the world within;

But can you get inside my head and see the world without?

That, Sir, is the sixty-four thousand dollar question. I fear the answer is "No."

ANDREW BAMJI

Middlesex Hospital,

'Blackwell P. Guy's Hospital Reports 1965;114: 2 Wade CC. Med Care 1965;3:189-92.

${ }_{*}^{*}$ This correspondence is now closed.-ED, $B M \mathcal{B}$.

\section{Treatment of sciatica}

SIR,-In reply to Dr Michael Snaith's letter (1 November, $p$ 1217), what is classical sciatica? I was taught that sciatica is a pain in the lower limb in the distribution of the sciatic nerve. This contains elements from the nerve roots of L4, L5, S1, and S2. The first three nerve roots can be compressed by a lumbar intervertebral disc, but they also produce the nerve supply to ligaments in the lower back and the sacroiliac joints and these ligaments, when strained, can refer pain to the dermatome of their nerve supply. It is a rarity for the S2 nerve root to be hit by a disc, but pain in the S2 dermatome-namely, at the back of the thigh and behind the knee-is a fairly common occurrence, this being caused by a strain of the sacrotuberous ligament. No investigation can prove a ligamentous cause of sciatica.

By my interpretation of the symptoms and signs on examination, the lumbar disc causes low back pain and sciatica in less than $50 \%$ of cases and only $1 \%$ require surgery.

London W1

RONALD BARBOR

\section{Hidden hypotheses}

SIR,-I have a great respect for both Professor J D Swales and Dr Mildred Seelig, and therefore read his distinguished review of her book on magnesium metabolism with much interest (25 October, $p$ 1132). The review itself was perfectly fair, but why has it become so fashionable for professors of medicine of positivist cast of mind to use any opportunity and the authority of Karl Popper to take a side swipe at psychoanalysis even when writing about mineral metabolism, or some other topic in their own field?

It would be my view that psychology found its Newton in Freud; but it took physics nearly 300 years to travel via Kant to Einstein and on the way it dallied for long periods with the kind of all-embracing explanatory concepts such as phlogiston and the ether that Professo Swales finds unacceptable in psychoanalysis and which may in fact have nourished rather than retarded its growth as a science. We do not find scientists such as the late Lord Adrian, working in fields closer to those tilled by psychoanalysts than Professor Swales's, sneering at their work, which like that of modern physicists needs to be relativistic and is subject to the uncertainty principle because the presence of the observer impinges so greatly on what is observed. Sooner or later psychology will find what Kant calls the true path of science and meanwhile it is properly concerned with the content as well as the forms of the mental processes of those suffering from psychiatric disorders-which are, like other diseases, experiments of nature able to throw light on normal function.

It was Freud himself who said that credulity was a necessary quality for the creative scientist and to be over-critical is, as Roy Campbell put it in relation to poetry, making bit and bridle more important than the horse. Perhaps seekers after the truth in any particular field of inquiry should like journeymen stick to their lasts; for, in the words of Hooker, labour involved in digging up in order to look at the philosophical roots of our assumptions is more necessary than pleasant.

University Department of Paediatrics,

JOHN DAvis Addenbrooke's Hospital

\section{Suppression of "rubral" tremor with levodopa}

SIR,-I have always considered that rubral tremor was present at rest and was exacerbated by movement when a cerebellar component was obvious. It is distinguished from a Parkinsonian tremor by being much more a flexion-extension movement of the wrist and fingers than the side-to-side pill-rolling tremor of Parkinsonism. It is not associated usually with paucity of movement or with cogwheel rigidity. In five patients whom I have treated deliberately with Sinemet or Madopar the response has been completely disappointing, and I was interested to see the report by Drs L J Findley and M A Gresty (18 October $p$ 1043) of a case in which levodopa seemed of some value in such a tremor. Perhaps the different response to drugs is due to an underlying variation of pathophysiology that produces the similar clinical picture.

Department of Neurology,

North Manchester General Hospital,
Manchester M8 6RB

\section{Electricity and bones}

SIR,-The 16 August (p 470) issue of $B M F$ has just come to my attention, and I was somewhat surprised to learn that "electrical treatment will, in time, become a useful addition to the orthopaedic armamentarium." This statement appears along with others equally perplexing in a leading article entitled "Electricity and bones." Apparently, you have not kept completely up to date with the latest developments in this rapidly expanding field. Furthermore, you make a mistake common to many neophytes in this new discipline of electrobiology.

Firstly, it is inappropriate to consider repair of fresh fractures and ununited fractures in common biological terms. One is an example of an acute repair process in which injury potentials and other factors trigger the formation of callus de novo. In the non-union, repair tissues already exist and are relatively quiescent. All of the evidence from animals and patients with non-unions treated with pulsing electromagnetic fields points to an evolution in the soft tissues (fibrocartilage and fibrous tissue) occupying the gap between the bone ends. No new callus has been observed during nonunion healing with pulsing electromagnetic fields. In fact, the mechanism involves mineralisation of the fibrocartilage, vascular penetration, removal of the provisionally "calcified material," and its replacement by bone. The process resembles closely normal endochondral ossification. Furthermore, recent studies have demonstrated that pulse characteristics which are highly effective in treating non-unions in the clinic have little or no effect on fresh fractures in animals. This specificity is in consonance with other in vitro and animal studies which have demonstrated selective biological actions of different pulse waveforms.

In the second mistake, the author has made little or no biophysical distinction between constant currents delivered by electrodes and pulsing currents induced in tissues by time-varying electromagnetic fields. From both physical and biological viewpoints effects of the two different systems may be quite different. This tendency to lump all electrical modalities together can only stultify our intellectual growth curve. It is as if we said that red and green are the same because both are colours.

Finally, I should like to bring to the attention of your readers an alternative view to the one expressed by your editorial-namely, "electrical treatment is now a useful addition to the orthopaedic armamentarium." In the programme with pulsing electromagnetic fields, the worldwide experience involves more than 2000 orthopaedic surgeons who have treated more than 3500 patients with this modality. In the first 1000 end results, the overall success rate is $80 \%$ for all categories of ununited bone discontinuities, including nonunions, failed fusions, and congenital "pseudarthroses." For tibial non-unions in adults, the success rate approaches $90 \%$ and compares most favourably with the best series involving operative repair. In many of these patients, particularly those with active infections and congenital "pseudarthroses," amputation has been avoided when al else has failed. Here in the United States the method has been approved as safe and effective by the Food and Drug Administration, and is universally available to the orthopaedic community.

I am afraid that many of your readers will interpret the tone of the leading article in such a way that the vast benefits of this breakthrough in controlling cell function may be denied to those who could benefit from its use. It would seem to me from reports reaching our shores that your country is beset by at least as many financial pressures on its economy as ours. Even if the risk factors were equal, the cost of a surgical repair, as opposed to outpatient care with pulsing electromagnetic fields, does not make good economic sense here, and may not in England. This takes into account your National Health Service system with a relatively fixed number of hospital beds and services. If the bed is occupied by a patient with non-union awaiting surgery, it cannot be occupied by a patient with osteoarthritis awaiting a hip prosthesis, or for that matter a patient with an acute myocardial infarction.

\section{ANDREW L BASSETT}

Department of Orthopedic

Department

College of Physicians and

Surgeons of Columbia

University,
New York, NY 10032, USA

${ }^{*} *$ Professor Bassett suggests that no proper understanding or reference is made in our leading article to the different types of electrical 